\title{
Acercamiento a la Discusión Académica sobre Blended Learning
}

\section{An approach to Academic Discussion about Blended Learning}

Perla del Refugio Escamilla-Martínez y Vanesa del Carmen Muriel-Amezcua ${ }^{2}$ \section{(2) EDICIÓN: Resiliencia}

Recibido: 10/julio/2021

Aceptado: 10/octubre/2021

P Publicado: 29/noviembre/2021

$\downarrow$ Páginas: $130-142$

\&aís

${ }^{1}$ México

${ }^{2}$ México

\section{IIIIInstitución}

${ }^{1}$ Universidad Autonóma de Querétaro ${ }^{2}$ Universidad Autonóma de Querétaro

\section{Correo Eletrónico}

1perla.esmtz@gmail.com 2vanesa.muriel@uaq.mx

\section{ORCID}

${ }^{1}$ https://orcid.org/0000-0002-2793-8272 ${ }^{2} \mathrm{https}: / /$ orcid.org/0000-0002-2020-7709

\section{Citar así: Lf APA / IEEE}

Escamilla-Martínez, P. \& Muriel-Amezcua, V. (2021). Acercamiento a la Discusión Académica sobre Blended Learning. Revista Tecnológica-Educativa Docentes 2.0, 1(1), 130-142 https://doi.org/10.37843/rted.v1i1.263

P. Escamilla-Martínez y V. MurielAmezcua, "Acercamiento a la Discusión Académica sobre Blended Learning", RTED, vol. 1, n. ${ }^{\circ}$ 1, pp. 130-142, nov. 2021.

\section{Resumen}

El Blended Learning, aprendizaje semipresencial o aprendizaje combinado es una modalidad de enseñanza que entrelaza componentes tanto de la presencialidad como de clases en línea. El presente artículo tuvo como objetivo presentar un bosquejo de los alcances, límites, retos así como de los vacíos del Blended Learning, a partir de una revisión de los trabajos publicados en los últimos seis años, por medio de un análisis interpretativo de los antecedentes, definiciones, beneficios, desafíos así como de sus obstáculos, el rol del docente en ambientes semipresenciales, modelos, medición de su efectividad. Este trabajo es una investigación de corte documental sustentada en el enfoque cualitativo interpretativo. Los hallazgos muestran un vacío en el consenso sobre una definición de dicha modalidad, se encuentra una coincidencia como determinante en capacitar a los profesores para implementar este modelo como principal desafío, además de una tendencia de medición de la efectividad de los modelos basada en el aprendiz, así como un enfoque predominante de corte constructivista para su implementación. Para concluir, otras líneas de investigación urgentes para el Blended Learning son aquellas relacionadas con sus fundamentos conceptuales, medición de su eficacia desde una perspectiva sistémica, una detección de necesidades de capacitación específica en habilidades y competencias tanto didácticas como tecnológicas para los docentes, específicamente en contextos híbridos.

Palabras clave: Aprendizaje semipresencial, enseñanza superior, tecnología de la información.

\section{Abstract}

Blended Learning is a teaching modality that intertwines face-to-face and online classes components. The objective of this article was to present an outline of the scope, limits, challenges as well as gaps of Blended Learning, based on a review of the works published in the last six years, through interpretive analysis of the antecedents, definitions, benefits, challenges as well as their obstacles, the role of the teacher in blended environments, models, measurement of their effectiveness. This work is documentary research based on the qualitative interpretive approach. The findings show a gap in the consensus on a definition of this modality; a coincidence is found as a determining factor in training teachers to implement this model as the main challenge. In addition, a trend of measuring the effectiveness of the models based on the learner, as well as a predominant constructivist approach to implementation. To conclude, other urgent lines of research for Blended Learning are those related to its conceptual foundations, measurement of its effectiveness from a systemic perspective, detection of specific training needs in both didactic and technological skills, and competencies for teachers, specifically in hybrid contexts.

Keywords: Blended learning, higher education, information technology. 


\section{Introducción}

El Blended Learning, aprendizaje semipresencial o aprendizaje combinado es una modalidad de enseñanza que entrelaza componentes tanto de la presencialidad como de clases en línea. Planteado como tal en los últimos años, resulta ser una opción viable para enfrentar los cambios en la educación superior, misma que exige una adaptación en la enseñanza a través de medios digitales, la cual, por razones educativas además de sociales, se considera una nueva forma de aprender y de enseñar, dado los beneficios que la semipresencialidad aporta a todos los agentes inmiscuidos en el proceso de educación.

Este nuevo tipo de ambiente de aprendizaje promueve una mejora de cuestiones tales como: calidad del aprendizaje, acceso, interacción entre los aprendices, además de reducir costos (CuestaMedina, 2018). Aunque históricamente en el aprendizaje mediado por tecnologías, ha sido común integrar tecnologías en ambientes físicos a través de varios métodos de aprendizaje, una particularidad esencial del Blended learning radica en su intencionalidad de combinar, articulando lo mejor de cada uno de ambos ambientes (Seraji, Attaran \& Azizi, 2019), siendo actualmente, el principal reto en su implementación. Esta modalidad que surge de las limitaciones identificadas para la puesta en práctica del e-learning para el abono en la adopción de nuevas metodologías de aprendizaje, alejando esta pretensión de una cuestión que enfatizaba la necesidad de requerimientos tecnológicos en aras de su éxito (Cabero-Almenara \& Llorente-Cejudo, 2008).

Más allá de los componentes técnicos que soportan a un aprendizaje en línea, se debate ahora sobre aspectos que rodean este esquema con una base conceptual desdibujada y el sistema de enseñanza distribuida, que pueden ser descritos tanto en el salón de clases como en aula virtual, enfatizando el rol central de la tecnología en cualquiera de ellos (Graham, 2004). Actualmente, el término de blended learning abarca muchas más dimensiones, de acuerdo con Singh (2003) estas combinaciones pueden ser: El aprendizaje fuera de línea y en línea, actividades a ritmo propio y en vivo con aprendizaje colaborativo, actividades estructuradas y desestructuradas, contenido personalizado y contenido fuera de la plataforma y el aprendizaje con la práctica y el apoyo al rendimiento.

Este artículo tuvo como objetivo presentar un bosquejo de los alcances, límites, retos así como de los vacíos del blended learning, a partir de una revisión de los trabajos publicados en los últimos seis años, por medio de un análisis interpretativo de los antecedentes, definiciones, beneficios, desafíos así como de sus obstáculos, el rol del docente en ambientes semipresenciales, modelos, medición de su efectividad, así mismo de teorías convergentes con el blended learning, todo soportado a través de una revisión de la literatura, la cual permita exponer un acercamiento al estado actual del conocimiento de dichas categorías con el fin de sustentar las bases para su implementación, mejora e innovación en el campo de la tecnología educativa.

\section{Metodología}

Este trabajo es una investigación de corte documental sustentada en el enfoque cualitativo interpretativo. Con base en Medina-Montoya (2004), dicho enfoque tiene el objetivo de efectuar una interpretación del material bibliográfico, a través de la lectura, revisión, extracción, además de un análisis interpretativo de la información de textos publicados.

A partir de categorías o unidades de análisis determinadas, las cuales fueron guía durante todo el proceso: antecedentes, definiciones, beneficios, desafíos, obstáculos, el rol del docente, modelos, medición de su efectividad así como de teorías de blended learning, además de una revisión lineal y transversal de la información, se realizó una comparación constante de categorías en las fuentes ordenadas, siendo posible su interpretación, la cual permitió, una identificación de vacíos, coincidencias, en 
suma, de ampliaciones del concepto. La población objeto de estudio estuvo constituida por los artículos publicados y propuestas desarrolladas sobre el blended learning, con énfasis en aquellos que contenían las categorías seleccionadas para el estudio en idioma español e inglés de los últimos seis años.

La recolección de información se basó en el proceso propuesto por Hoyos-Botero (2000) quien establece, en primera instancia, una preparación de la información en donde se definen el objeto de estudio, pasos, también etapas; seguido de una fase descriptiva, donde se establece el tipo de estudio, los referentes disciplinares, muestra, delimitación, de igual manera una metodología; como tercer paso se tiene una fase constructiva en la cual se identifican tendencias, logros, vacíos, limitaciones, también las dificultades de este modelo; seguido de una fase interpretativa, donde se proporcionan hipótesis interpretativas igualmente una ampliación del horizonte del estudio así como una construcción teórica global para finalmente proceder a la extensión y publicación (Londoño-Palacio, Maldonado-Granados, \& Calderón-Villafánez, 2016).

La preparación consistió en delimitar fuentes documentales para identificar vacíos y también hallazgos de acuerdo con las categorías: antecedentes, definiciones, ventajas, desafíos, obstáculos, teorías así como modelos de blended learning, esta delimitación fue llevada a cabo mediante una búsqueda en las siguientes bases de datos: Jstor, Dialnet, Redalyc y Scielo, su elección se justifica dado el volumen de información de acceso libre, la cual hace referencia en su contenido o título sobre el blended learning .

Dentro de la fase descriptiva se delimitó el corpus de la investigación, tomando en cuenta los criterios de fecha de publicación entre los años 2014 y 2020 con coincidencia con las categorías mencionadas referidas al nivel de Educación Superior en el idioma inglés y español, la búsqueda se realizó usando descriptores como blended learning , blended learning, hybrid learning, blended learning higuer education, blended learning models, semipresencialidad, aprendizaje combinado y modelos de blended learning ; después de la selección de los artículos se precedió a la siguiente fase.

La fase constructiva fue realizada mediante una matriz analítica de contenido, se realizó un análisis de cada texto y se extrajeron los fragmentos relacionados con las categorías en fichas analíticas, donde se plasmaron observaciones de cada texto con respecto a los datos de la fuente del artículo, propósito, ideas centrales, palabras clave y conclusiones del mismo, de esta forma, después de organizar la información, los textos de la muestra fueron relacionados verticalmente mientras las categorías de análisis horizontalmente; se describieron los hallazgos para proceder a una construcción de sentido de los mismos (Baeza-Correa, 2008), siendo este proceso el correspondiente a la construcción teórica global. A partir de ahí se procedió a ampliar el contenido seguido de la identificación de vacíos, coincidencias hasta la redacción de los resultados.

\section{Resultados}

A continuación se presentan los resultados del análisis interpretativo de los artículos que por guardar relación con los criterios y categorías fueron seleccionados para construir la revisión de la literatura sobre blended learning, se encontró una falta de consenso sobre la definición del blended learning, una coincidencia en la importancia de la capacitación docente para la funcionalidad de este modelo organizacional en las instituciones, así mismo un enfoque de evaluación de efectividad de modelo basado en su mayoría en su impacto en el aprendiz, agregado a lo anterior, se encontró una coincidencia en el enfoque constructivista como fundamento teórico predominante.

Los resultados principales del análisis por categoría se muestran en Tabla 1, se enuncian los hallazgos, el autor donde se menciona y el año, seguido de esto se describen de manera específica. 
Tabla 1

Elementos Encontrados en la Definición de Blended Learning

\begin{tabular}{|c|c|c|c|}
\hline Autor & Año & $\begin{array}{l}\text { Denominación de la } \\
\text { modalidad presencial }\end{array}$ & $\begin{array}{l}\text { Denominación } \quad \text { de } \\
\text { modalidad no presencial }\end{array}$ \\
\hline Prohorets y Pleckhanova & 2014 & Modalidad face to face & $\begin{array}{l}\text { Un sistema de enseñanza } \\
\text { distribuida }\end{array}$ \\
\hline $\begin{array}{l}\text { Holdenn y Duhane, en } \\
\text { Wengreen, Dimmick e } \\
\text { Israelsen. }\end{array}$ & 2015 & Modalidad cara a cara y una & Instrucción en línea \\
\hline Cuesta-Medina & 2018 & Comunicación síncrona y física & Virtual (síncrona o asíncrona) \\
\hline
\end{tabular}

En la Tabla 2 se muestran los beneficios que los autores mencionan acerca de la modalidad blended learning, las áreas de beneficios son principalmente en cuanto a la autonomía y flexibilidad en el estudiante así como el logro de nuevas competencias.

Tabla 2

Beneficios en el Uso de Blended Learning

\begin{tabular}{|c|c|c|c|}
\hline Autor & & Año & Área de beneficio \\
\hline $\begin{array}{l}\text { Cabero } \\
\text { Román }\end{array}$ & $\mathbf{y}$ & 2008 & El aprendizaje autónomo \\
\hline $\begin{array}{l}\text { Martín, } \\
\text { Serrani } \\
\text { Gómez }\end{array}$ & $\mathbf{y}$ & 2014 & El profesorado \\
\hline $\begin{array}{l}\text { Johnson, } \\
\text { Becker, } \\
\text { Estrada } \\
\text { Freeman }\end{array}$ & $\mathbf{y}$ & 2015 & $\begin{array}{l}\text { El logro de nuevas } \\
\text { competencias }\end{array}$ \\
\hline $\begin{array}{l}\text { Johnson, } \\
\text { Becker, } \\
\text { Estrada } \\
\text { Freeman }\end{array}$ & $\mathbf{y}$ & 2017 & $\begin{array}{llr}\text { Flexibilidad } & \text { en } & \text { el } \\
\text { aprendizaje } & \text { de } & \text { los } \\
\text { estudiantes. } & & \\
\text { s. } & & \end{array}$ \\
\hline $\begin{array}{l}\text { Rodríguez } \\
\text { Espinoza }\end{array}$ & $\mathbf{y}$ & 2017 & Programa académico \\
\hline $\begin{array}{l}\text { Gallou } \\
\text { Abrahams }\end{array}$ & $\mathbf{y}$ & 2018 & $\begin{array}{l}\text { El aprendizaje basado en la } \\
\text { investigación, } \\
\text { multidisciplinario } \\
\text { interdisciplinario }\end{array}$ \\
\hline
\end{tabular}

Nota. Los beneficios del blended learning de acuerdo con autores, elaboración propia (2021).

En la tabla 3 se enlistan las áreas de desafío y obstáculos para implementar el blended learning mencionados por los autores, destacan el soporte a profesores para que conozcan como implementarlas, así como la falta de formación en los mismos.

\section{Tabla 3}



Simón, Benedi, 2018 Falta de tiempo, Blanché, Bosch y habilidades Torrado pedagógicas y de formación de los profesores.

\begin{tabular}{lcl}
\hline Serrano y Gómez & 2015 & $\begin{array}{l}\text { Desconocimiento } \\
\text { de los profesores }\end{array}$ \\
\hline Trentin & 2015 & $\begin{array}{l}\text { Contexto } \\
\text { didáctico- } \\
\text { pedagógico }\end{array}$ \\
\hline Gallou y Abrahams & 2018 & $\begin{array}{l}\text { Soporte } \\
\text { profesores }\end{array}$
\end{tabular}

Martín García, 2014 Postura de los
Serrano y Gómez gestores de las universidades frente a las TIC

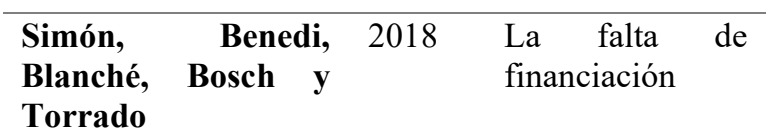

Nota. Áreas de desafío y obstáculos para implementar el blended learning, elaboración propia (2021).

Cuando se implementa una modalidad blended learning surgen algunas necesidades, algunos autores mencionan que son necesarios modelos de actuación para incorporar diversidad de tipos de aprendizaje, la necesidad de realizar estudios que consideren al docente y a la institución, así como la incorporación y normalización del uso de las tecnologías en la práctica docente (ver Tabla 4). 


\section{Tabla 4}

El Rol del Docente para la Implementación del Blended Learning

\begin{tabular}{|c|c|c|c|}
\hline Autor & & Año & $\begin{array}{l}\text { Necesidades } \\
\text { encontradas }\end{array}$ \\
\hline $\begin{array}{l}\text { Zabala } \\
\text { Arnau }\end{array}$ & $\mathbf{y}$ & 2014 & $\begin{array}{l}\text { Modelos de actuación } \\
\text { para incorporar diversos } \\
\text { tipos de aprendizaje }\end{array}$ \\
\hline $\begin{array}{l}\text { Wang, Han } \\
\text { Yang }\end{array}$ & $\mathbf{y}$ & 2015 & $\begin{array}{l}\text { Realizar estudios que } \\
\text { consideren el impacto de } \\
\text { la modalidad blended } \\
\text { learning también en el } \\
\text { docente y la institución }\end{array}$ \\
\hline $\begin{array}{l}\text { Cabero, Roig } \\
\text { Vila } \\
\text { Mengual }\end{array}$ & $y$ & 2017 & $\begin{array}{l}\text { Combinar factores } \\
\text { tecnológicos con los } \\
\text { contenidos disciplinares }\end{array}$ \\
\hline Been & & 2018 & $\begin{array}{l}\text { Normalizar el uso de las } \\
\text { tecnologías en el } \\
\text { quehacer docente }\end{array}$ \\
\hline $\begin{array}{l}\text { Núñez, } \\
\text { Monclúz } \\
\text { Ravina }\end{array}$ & $\mathbf{y}$ & 2019 & $\begin{array}{l}\text { Adecuación de } \\
\text { estrategias docentes para } \\
\text { los requerimientos } \\
\text { sociales y laborales }\end{array}$ \\
\hline
\end{tabular}

Nota. Consideraciones del docente y a la institución, elaboración propia (2021).

Existen diversos modelos a los cuales recurrir para implementar la modalidad blended learning, en la Tabla 5 se mencionan los encontrados en esta revisión de la literatura.

\section{Tabla 5}

Modelos y Medición de la Efectividad del Blended Learning

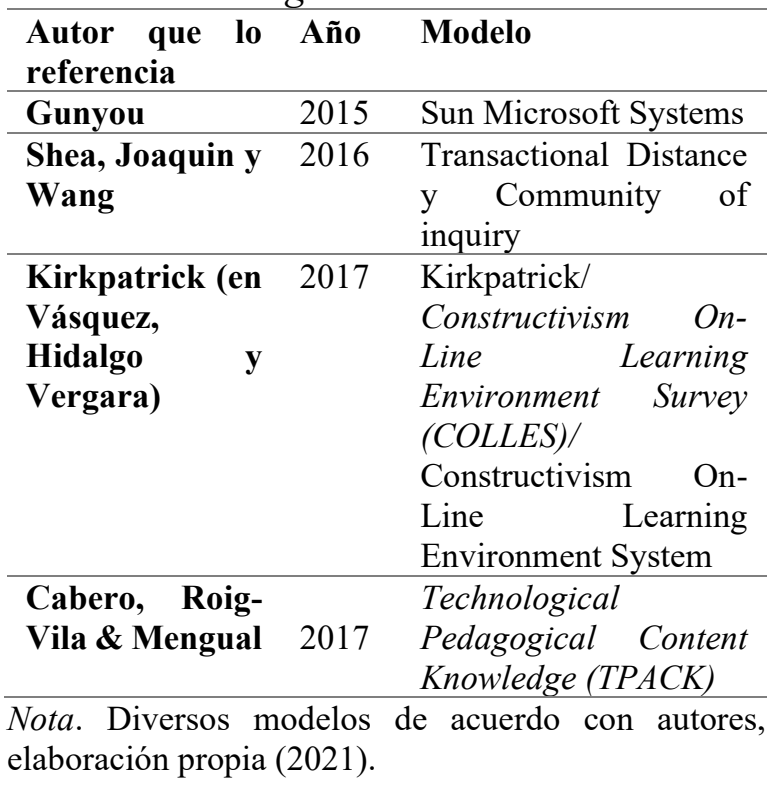

Las teorías en las que más coinciden los autores en las que se fundamenta esta modalidad (ver Tabla 6) son principalmente el constructivismo, conectivismo $\mathrm{y}$ cognitivismo.

\section{Tabla 6}

\begin{tabular}{|c|c|c|c|}
\hline Autor & & Año & Paradigma Teórico \\
\hline $\begin{array}{l}\text { Chris } \\
\text { Moldovan, } \\
\text { Murphy, } \\
\text { Pathak } \\
\text { Muntean }\end{array}$ & $\mathbf{y}$ & 2018 & Constructivismo \\
\hline $\begin{array}{l}\text { Solorzano } \\
\text { García, }\end{array}$ & $\&$ & 2018 & Conectivismo \\
\hline Sobrino & & 2014 & $\begin{array}{l}\text { Modelo Pedagógico } \\
\text { Poscontructivista }\end{array}$ \\
\hline $\begin{array}{l}\text { Cuevas, } \\
\text { Feliciano, } \\
\text { Miranda } \\
\text { Catalán }\end{array}$ & $\mathbf{y}$ & 2015 & $\begin{array}{l}\text { Identificación de } \\
\text { componentes } \\
\text { conductismo, } \\
\text { cognitivismo, } \\
\text { transmisión-recepción y } \\
\text { descubrimiento guiado }\end{array}$ \\
\hline
\end{tabular}

Nota. Teorías Blended Learning según autores, elaboración propia (2021).

\section{Definición de Blended Learning}

El aprendizaje combinado tuvo sus inicios en el ámbito empresarial con una orientación hacia la formación de recursos humanos, la práctica de esta modalidad fue trasladada al campo educativo iniciando principalmente con el uso de páginas web y plataformas, ambos recursos de una naturaleza estática en términos de virtualidad, posteriormente se empiezan a introducir componentes de colaboración y comunicación entre los usuarios (Vázquez, Hidalgo \& Vergara-Lozano, 2017). Desde entonces varios enfoques han sugerido una visión y conceptualización del término a modo muy amplio, dentro de tal ambigüedad, otras modalidades podrían ser incluidas dentro del aprendizaje combinado

La falta de precisión de un concepto del aprendizaje combinado puede traer consecuencias en su ejecución además en sus resultados, al intentar implementar una modalidad de aprendizaje mixta la cual desde su concepción resulta ser más bien e-learning, enseñanza abierta, móvil u otra, conduce a una interpretación errónea de sus beneficios y por lo tanto de su transferencia.

$\mathrm{Su}$ adopción en el ámbito académico fue aumentando a partir del año 
2002, cuando más investigación acerca de esta práctica comenzó a documentarse y publicarse, aunque el término en sí había sido utilizado desde décadas antes; una cuestión importante de esta modalidad en su mayoría fue esclareciéndose como una combinación de dos métodos de instrucción o dos medios de entrega, aunque otros conceptos relacionados han surgido como sinónimos, tales como el aprendizaje híbrido o mixto, el aprendizaje combinado ha predominado al referirse a dos tipos de instrucción o medios combinados.

En su traducción literal el "blended learning" está referido al aprendizaje "mezclado" pues el término blended proviene del verbo to blend el cual en español se traduce como mezclar o combinar, de ahí han surgido otras denominaciones como: bimodal, híbrido, combinado, semipresencial, integrado, dual, entre otros (García-Aretio, 2020, p.12). Actualmente esta concepción no se limita únicamente a una combinación de modalidades en línea con presenciales, se trata más de una combinación de recursos, medios, modalidades, técnicas, así como actividades las cuáles potencialicen las bondades de cada una de ellas en un entorno flexible de aprendizaje.

Los conceptos de blended learning deben ser revisados para tener un entendimiento claro de su significado, por otro lado, conocer si las estrategias utilizadas por los diversos actores educativos realmente corresponden con este modelo. Algunos autores como Prohorets \& Plekhanova (2014), lo definen como una combinación entre una enseñanza cara a cara y un sistema distribuido, enfatizando el rol de la tecnología en ambos.

El carácter del sistema cara a cara puede entenderse como los acercamientos de persona a persona ya sea de manera virtual o presencial, el sistema de enseñanza distribuida alude a aquellas actividades la cuales el discente es capaz de desarrollar a su propio ritmo, en donde tanto los contenidos como actividades están en un entorno virtual, en este sentido, actualmente el blended learning puede combinar una o más dimensiones que van más allá de su concepción original como simplemente vincular el salón de clases tradicional con actividades en línea.

También se le conoce como aprendizaje combinado al referirse a la integración de la instrucción tradicional cara a cara con una instrucción en línea, en la cual, los estudiantes pueden tener algún grado de control sobre el tiempo, lugar o ritmo del aprendizaje (Wrengreen, Dimmick e Israelsen, 2015). Otras tendencias apuntan a considerar una ampliación de enfoques dentro de su concepto, para evitar una confusión conceptual existente del concepto blended learning para de este modo, clarificar la medida con la cual puede ser considerada una innovación educativa, previniendo así caer en el error de considerar el uso de una plataforma virtual en apoyo al aprendizaje presencial como una metodología combinada (Martín, Serrano \& Gómez, 2014; Spring, Graham \& Hadlock, 2016).

De manera similar, se considera que los ambientes híbridos son dinámicos, creados por un movimiento constante de usuarios, en este caso los estudiantes, quienes llevan dispositivos móviles o portables y que están constantemente conectados a internet así como a otros usuarios, donde una parte de la comunicación tiene lugar tanto de manera síncrona como física mientras otra tiene lugar de manera virtual, esta puede ser síncrona o asíncrona, haciendo de este modo una contribución a la mejora en las interacciones creando así un ambiente de aprendizaje más amigable (Cuesta-Medina, 2018; Trentin, 2016).

\section{Beneficios del Blended Learning}

En cuanto a los beneficios del Blended learning algunos autores lo consideran como una modalidad óptima tanto para el aprendizaje como para el logro de nuevas competencias, así mismo, es establecido como un modelo que mejora el proceso de aprendizaje de los estudiantes porque cuenta con ventajas metodológicas sobre la mejora de la enseñanza tradicional e igualmente proporciona mayor flexibilidad a los estudiantes (Johnson, Becker, Estrada \& 
Freeman, 2015; 2017), una de sus mayores ventajas es la posibilidad de otorgar beneficios para el programa académico.

Esta metodología se caracteriza por promover un aprendizaje más autodidacta, colaborativo, de la misma forma, de resolución de problemas al combinar aspectos de dos modalidades, se pueden rescatar estrategias de cada una que favorezcan la efectividad del proceso de enseñanza aprendizaje, su flexibilidad, además de una fidelidad de la interacción, una modalidad mixta con aplicación efectiva radica en una adecuada asignación del tiempo cara a cara así como de instrucción en línea o distribuida, las decisiones de combinación de ambientes, tiempo y fidelidad son determinantes para definir dimensiones de interacción en un ambiente de aprendizaje combinado.

Por otro lado, se encuentran pocos los estudios abordando las ventajas de esta modalidad en otros aspectos como los costos de operación, ahorro de tiempo, recurso humano, inversión e infraestructura, los cuáles también deberían ser estudiados de manera más amplia y profunda, como beneficios esenciales (Rodríguez \& Espinoza, 2017). Adicionalmente, el aprendizaje combinado constituye ventajas para el profesorado cuando cuenta con una formación sobre el uso y aplicación de este modelo, teniendo como beneficios: reducción de la incertidumbre respecto a la innovación, mejora de actitud y atención en los estudiantes al ser implantado (Martín, Serrani \& Gómez, 2014).

Aunado a esto, Gallou \& Abrahams (2018) plantean a la virtualidad como apoyo a clases presenciales como una modalidad capaz de traer mejoras en algunos tipos de aprendizaje tales como el basado en la investigación, multidisciplinario e interdisciplinario, estos tipos de aprendizaje conceden a los estudiantes el desarrollo de habilidades esenciales para lograr ser parte activa dentro de proyectos, incluso puede ser útil para vincular a los investigadores con los estudiantes proveyendo experiencias de tipo multisensorial para adquirir información en el proceso de enseñanza, sirviendo así como incubadora para crear conexiones entre distintas disciplinas, agregando a lo anterior, Cabero \& Román (2008) señalan un aspecto mencionado como ventaja del blended learning se refiere a la descolocación del conocimiento, así como una autonomía que se desarrolla en el estudiante al permitir una formación just in time y just for me.

\section{Desafíos y Obstáculos del Blended learning}

En otro orden de ideas, implementar un sistema de enseñanza aprendizaje semipresencial involucra prever inconvenientes respecto a características del contexto educativo las cuales puedan evitar repercusiones en la calidad de la interacción profesor - alumno, estas fallas son principalmente resultantes de aspectos institucionales que van desde una ambigüedad o falta de claridad de las tipologías de las modalidades de enseñanza en línea, en particular de una imprecisión en la concepción del blended learning, así como también de sus fundamentos teóricos hasta aspectos tecnológicos y de formación docente para esta modalidad (Martín-García, Serrano \& Gómez, 2014; Simón, Benedi, Blanché, Bosh \& Torrado, 2018).

Por otro lado, existen aspectos más específicos del contexto educativo los cuales limitan el hecho de una implementación de esta modalidad, entre los cuales están englobados componentes asociados con políticas internas institucionales tales como una excesiva carga de trabajo del docente, lo cual repercute en una falta de tiempo para preparar de contenidos en línea los cuales complementen sesiones cara a cara, así como poca o nula capacitación en competencias digitales docentes, por ende se deriva un desconocimiento para una adecuada aplicación de esta innovación por parte de los mismos (Martín, García, Serrano \& Gómez, 2014; Torrado, 2018).

La capacitación docente es por consiguiente, uno de los factores cruciales para ser reforzado dentro de las instituciones, para brindar un soporte a los profesores en cuanto al enfoque de enseñanza y el uso de herramientas tecnológicas hasta dispositivos para el logro de una implantación exitosa de 
una modalidad híbrida, en consecuencia, esto puede desarrollar un papel fundamental para mejorar tanto la enseñanza como el aprendizaje de los estudiantes a través del aprovechamiento de estas herramientas; en otras palabras, la figura del profesor no debe ser reemplazada en un entorno híbrido pero si soportada a través de crear entornos virtuales a la vez de hacer uso de herramientas como foros, correos electrónicos, blogs, twitter y plataformas educativas (Gallou \& Abrahams, 2018; Núñez-Barriopedro, Monclúz， \& Ravina-Ripoll, 2019).

También se hace necesario capacitar a los docentes en metodologías didácticas las cuales propondrán los profesores a sus estudiantes, enfatizando el uso habitual y metódico de las TIC, no sólo es cuestión de disponibilidad tecnológica sino también de su razón de uso así como de la forma en cómo se usará para potencializar y revolucionar los procesos de enseñanza-aprendizaje, sobre todo el hecho de brindar un ambiente híbrido considerando su adaptabilidad al contexto didáctico/pedagógico (Trentin, 2016).

\section{El Rol del Docente en la Semipresencialidad}

En gran parte de la literatura se identifica un importante rol del docente para la implementación de un sistema híbrido de aprendizaje, se destaca un enfoque de enseñanza predominante en el constructivismo así mismo el reconocimiento por parte de los profesores tanto de incluir de manera urgente tecnologías de la información en su práctica docente, como de adecuar estrategias para una educación superior capaz de adaptarse a los requerimientos sociales y laborales (Núñez-Barriopedro, Monclúz \& Ravina-Ripoll, 2019), por otro lado, se destaca el imperativo de incorporar modelos de actuación que involucren los diversos tipos de aprendizaje tales como el aprendizaje activo, colaborativo, reflexivo, constructivista y autónomo (Zabala \& Arnau, 2014).

La tecnología como apoyo de las clases presenciales tradicionales supone ser una pieza que coadyuva a facilitar una mayor interacción del docente con los estudiantes, al respecto se hace necesario su utilización por parte de los profesores complementadas con una pedagogía capaz de articular los factores tecnológicos así como los contenidos disciplinares con un nivel óptimo de competencias docentes de modo que esta combinación se convierta en una parte normal del quehacer docente (Cabero, Roig-Vila \& Mengual, 2017; Been, 2018).

En una investigación en donde se analizaron 87 estudios empíricos sobre la literatura de blended learning, realizado por Wang, Han \& Yang (2015), se encontró que una gran parte de los artículos están centrados en el estudio del aprendiz o estudiante, seguido del contenido y la tecnología. Los porcentajes caen cuando se refieren a los docentes, la institución y al soporte para el aprendizaje. Los resultados presentados, permiten dar cuenta de una necesidad de un enfoque que no considere únicamente su efectividad en el aprendizaje del alumno, sino también considere sus implicaciones e impacto tanto en el docente como en la institución tomando en cuenta la tecnología, contenidos y soportes disponibles para el aprendizaje para su adecuación al sistema de manera multifactorial en los subsistemas de su contexto.

\section{Modelos y Medición de su Efectividad}

Un ambiente virtual de enseñanza cuenta con diversos modelos para su implementación los cuales, de manera gradual, puedan asegurar el acompañamiento y respuesta al estudiante. Entre los principales modelos para la modalidad blended learning encontrados en la revisión de la literatura, destacan los siguientes: el Content Support Model basado en la incorporación de contenidos casi sin cambios, similar a un inventario de materiales del curso; el Wrap Around Model, el cual consiste en un material hecho a la medida como guías de estudio y actividades de discusión; el Integrated Model, consiste en actividades colaborativas, recursos de aprendizaje y actividades de articulación (Breen, 2018).

Por un lado, los modelos Transactional Distance y Community of Inquiry, se ocupan de reducir la distancia psicológica en el 
contexto de aprendizaje, se afirma que hasta ahora existe poca exploración para averiguar una forma en la cual los instructores puedan combinar ambos modelos para asegurar tanto la satisfacción como el diseño para de esta manera, generar un alto grado de desempeño (Shea, Joaquin \& Wang, 2016)

En relación con su efectividad, uno de los factores más investigados en los sistemas de aprendizaje combinado de acuerdo a un estudio realizado en la Universidad Agraria del Ecuador por Vásquez, Hidalgo \& Vergara (2017) quienes utilizaron la plataforma Moodle siguiendo el modelo de formación Kirkpatrick, el cual contempla cuatro aspectos para su análisis: las reacciones, transferencia, aprendizaje e impacto, recurrieron para su estudio a un instrumento desarrollado por Taylor \& Maor llamado COLLES (Constructivism On-Line Learning Environment Survey); esta es una encuesta sobre el ambiente constructivista en línea el cual examina las dimensiones de relevancia, reflexión, interactividad, apoyo de los tutores, apoyo de los compañeros e interpretación (Vázquez, Hidalgo \& Vergara, 2017). En cuanto a efectividad, Strauss, Shanley, Lytell, Crowley, Bana, Megan \& Leuschner (2013), afirman que para la efectividad de un programa en línea deben proyectarse estrategias instruccionales de tal modo que ese diseño sea compatible con el proceso de aprendizaje humano.

Por su parte, Garrison, Anderson \& Archer desarrollaron el modelo COI (Community of Inquiry) el cual ha sido relevante porque permite explorar ambientes virtuales de aprendizaje, pero no se encuentra evidencia de que sea apto para ambientes híbridos (Wang, Han \& Yang, 2015). Por otra parte, Cabero, Roig-Vila \& Mengual (2017), apoyan el uso del modelo TPACK (Technological Pedagogical Content Knowledge) el cual describe una confluencia necesaria entre lo tecnológico, pedagógico, contenido y conocimiento, bajo la premisa de una imposibilidad de separar estos tres porque sus intersecciones generan siete tipos de conocimientos: conocimiento disciplinar, conocimiento pedagógico, conocimiento tecnológico, conocimiento pedagógico disciplinar, conocimiento tecnológico disciplinar, conocimiento tecnológicopedagógico y conocimiento tecnológico, pedagógico y disciplinar.

Otros modelos como el CABLS (Complex Adaptive Blended learning System) parten de identificar vacíos en la investigación y prácticas existentes sobre el aprendizaje combinado, este modelo de seis dimensiones: el maestro, la institución, el soporte de aprendizaje, el aprendiz, el contenido y la tecnología, sugiere identificar una interdependencia e interacción dinámica entre los subsistemas de estos componentes, lo cual marca una diferencia de este modelo respecto a los modelos existentes pues ninguno ha sido capaz de proveer un panorama completo de aprendizaje combinado ni de explorar a éste como un enfoque de sistema adaptativo complejo.

El modelo anterior está basado en la teoría de sistemas adaptativos complejos propuesta por Cleveland en 1994, ha sido ampliamente usado para lograr un entendimiento de la dinámica de los sistemas no lineales y abiertos los cuales intercambian materia e información al cruzar sus fronteras, usan ese intercambio de energía para mantener su estructura. Es capaz de promover una visión holística y sistemática del aprendizaje híbrido para poder proveer una ilustración completa de este aprendizaje, a diferencia de otros modelos que ignoran sus cualidades dinámicas (Wang, Han \& Yang, 2015).

El mayor debate entre los modelos se da alrededor de su efectividad pedagógica, sin embargo, una de las mayores ventajas del blended learning es su potencialidad de adaptar diferentes estilos de aprendizaje. Existen otros modelos como el de Gill Salmón en donde el moderador sigue una serie de etapas para producir en el estudiante una sensación de comodidad al introducido paulatinamente en un ambiente virtual, para otros autores ha sido considerado como uno de los modelos con consistencia en la jerarquía de necesidades de Maslow. Incorporar el blended learning en el nivel universitario puede valerse de este modelo para que de manera paulatina sea aceptado 
con los resultados esperados y sea capaz de ampliar de manera paulatina la experiencia de los estudiantes.

Otro modelo es el de Sun Microsoft Systems basado en ecologías de aprendizaje el cual toma forma de un cuadrante con aprendizaje autodirigido/aprendizaje, así como también vertientes de contenido/práctica, una desventaja de este modelo es el hecho de no expresar claridad en su implementación. Por otro lado, se encuentra la modalidad de Aula Invertida, esta une exitosamente lo mejor de las clases presenciales con las clases en línea, al introducir flexibilidad al aprendizaje, mientras conserva la eficacia del contacto institucional directo, de acuerdo con Gunyou (2015) debería ser impuesto como un método a seguirse en las instituciones. En ese sentido, es crucial identificar modelos los cuales puedan ser implementados tomando en cuenta su pertinencia con condiciones educativas propias del centro educativo, así como de competencias didáctico-tecnológicas de los docentes específicamente para entornos híbridos.

\section{Teorías}

Aunque el blended learning está soportando en diversas teorías como el conductismo, cognitivismo y humanismo, se encuentra una predominante inclinación por el paradigma constructivista para su implementación estableciendo el hecho de la construcción del conocimiento de las personas a través de su propio entendimiento del mundo, se constituye como un enfoque capaz de proveer a los estudiantes oportunidades para el uso de técnicas activas de aprendizaje como la discusión, colaboración y reflexión de aprendizaje en entornos híbridos (Chris Moldovan, Murphy, Pathak \& Muntean, 2018).

Una corriente contemporánea es el conectivismo, presentado por George Siemens (2004), la cual considera el conocimiento como distribuido a través de redes de conexiones en las cuales el aprendizaje puede incurrir a partir de atravesar esas redes, de acuerdo con esto, el rol del docente cambia a organizador del aprendizaje, facilitando a los estudiantes estrategias y herramientas utilizando nodos para formar una red, dichos nodos pueden ser personas u otros ambientes tanto educativos como no educativos, bases de datos, revistas, libros o pueden ser algunas otras fuentes de información (Solórzano-Martínez \& GarciaMartinez, 2016).

Con la teoría del conectivismo queda de manifiesto una percepción del papel de la tecnología en la educación, al ser vista como una red de conocimiento donde se empiezan a redefinir todos los fundamentos los cuales permitirían una generación de conocimiento en esta era digital, de tal modo que podrían superar los modelos anteriores. En ese sentido, Sobrino (2014) considera al blended learning como un modelo pedagógico postconstructivista pues de acuerdo con el autor, los modelos teóricos anteriores no tienen en cuenta el aprendizaje producido fuera de los individuos, ni son capaces de describir cómo ocurre el aprendizaje almacenado y manipulado por la tecnología, a diferencia del constructivismo, establece que el alumno debe conferir un significado a través de la comprensión al ejecutar tareas, en el conectivismo este significado ya existe.

Aun así, hay quienes afirman que, para poder referirse al aprendizaje combinado, se debe mirar a través del constructivismo, no obstante, tienen que ser identificados los componentes pertenecientes a otras teorías o modelos tales como: el conductismo, cognitivismo, transmisión-recepción $\mathrm{y}$ descubrimiento guiado (Cuevas, Feliciano, Miranda \& Catalán, 2015). Dadas las conceptualizaciones estas teorías, se puede observar una tendencia post constructivista, aun cuando el constructivismo ha sido la base de los modelos semipresenciales de enseñanza, este también debe retomar aspectos de otras nuevas teorías del aprendizaje, dando a entender que un solo enfoque sería insuficiente para su implementación y adaptación.

\section{Discusiones}


Dentro del análisis de la primera categoría revisada en el estado del arte sobre la definición de blended learning, mientras algunos lo definen como una combinación de una modalidad face to face y un sistema de enseñanza distribuida, otros hacen alusión a esta distribución como la "modalidad en línea" como complemento a una enseñanza cara a cara, esta última apunta a crear confusión, esto porque se puede cometer el error de usar una plataforma virtual como complemento y considerar esto como blended learning. Así mismo otros autores lo conciben como una comunicación síncrona y física con otra virtual (síncrona o asíncrona). También se encuentran una diversidad en de denominaciones del concepto blended learning tales como: "blended learning", "aprendizaje híbrido" y "espacios híbridos".

En cuanto a las ventajas del blended Learning, gran parte de los autores coinciden en su eficacia para mejorar el proceso de enseñanzaaprendizaje. Se identifica en gran parte de los textos analizados estas ventajas desde una perspectiva de los beneficios para el programa académico, así como pocos los estudios que consideran otros aspectos ventajosos como los costos de operación, ahorro de tiempo, recurso humano, inversión e infraestructura, aunado a esto, se encuentran grandes ventajas para el profesorado, el aprendizaje multidisciplinario e interdisciplinario y el aprendizaje basado en la investigación.

En la categoría de desafíos y obstáculos, se pueden identificar dificultades asociadas con el profesorado tales como: falta de tiempo, de habilidades pedagógicas, de formación, técnicas y desconocimiento de las implicaciones de la modalidad blended learning; por otro lado, encontramos dificultades asociadas con el contexto didáctico-pedagógico, tales como: el soporte a profesores, posturas de los gestores de las universidades, así como la falta de financiación. A partir de lo anterior, se pueden identificar dos grandes áreas que obstaculizan la implementación de esta modalidad: las referentes al profesorado y al contexto didáctico-pedagógico; ambas indican una necesidad de reestructuración de políticas internas de cada institución educativa para brindar apoyo a los tutores para de este modo implementar eficazmente el modelo blended learning.

Como se identifica en las categorías anteriores, uno de los componente más destacado del contexto educativo para la eficacia de esta modalidad es el rol del docente, sin embargo, en su mayoría, las investigaciones sobre blended learning tal como afirman Wong, Han \& Yan (2015) están más centrados en otros aspectos así como en una confirmación de una necesaria capacitación docente para una incorporación de recursos tecnológicos, aunado a una pedagogía y contenidos disciplinares en la práctica y el desarrollo de competencias docentes.

En relación con los modelos y medición de la efectividad del blended learning, los modelos más utilizados son: Content-Support Model, Wrap Around Model y el Integrated Model; modelos que se establecen para medir variables como la satisfacción y el diseño del curso tales como el Transaccional Distance y el Community of Inquiry; también existen otros modelos más enfocados en la medición de la efectividad como el Kirkpatrick con la escala COLLES (Constructivism On Line Learning Environment Survey); y otros con un enfoque más holístico que tienden a considerar otros aspectos como lo pedagógico y de contenido: TPACK (Technological Pedagogical Content Knowledge) y los subsistemas del contexto como el CABLS (Complex Adaptive Blended learning System); así como modalidades de blended learning con gran aceptación por sus resultados como el Aula Invertida.

Finalmente se puede establecer en la revisión teórica, de acuerdo con el consenso en la revisión de la literatura, los fundamentos de esta modalidad son muy similares a aquellas que soportan los ambientes de aprendizaje virtuales, en el caso de blended learning, el enfoque predominante actual es el constructivismo, no obstante, se identifica una necesidad de complementarse con fundamentos de otras teorías como el conectivismo.

\section{Conclusiones}


El blended learning es una modalidad la cual trae consigo una amplia gama de beneficios al proceso de enseñanzaaprendizaje, ciertamente se percibe aún una confusión conceptual reflejada en su interpretación, así como también en su aplicación alejada de su propósito central, el cual consiste en el aprovechamiento tanto de los recursos presenciales como no presenciales. Asociado a esto, se encuentran obstáculos de tipo institucional, de capacitación en habilidades tales como competencias digitales docentes de modo que resultan ser los retos principales, así como prioritarios para su implementación, agregando a lo anterior, el rol del docente es fundamental para su eficacia, esto debido a que en la medida en que estén mejor capacitados, pueden contribuir a la mejora de su operabilidad.

Los modelos que fundamentan esta modalidad enfatizan sólo algunos de los componentes del contexto educativo, principalmente al aprendiz, si bien es el objetivo de cualquier modelo, existen aún esfuerzos por enfoques para considerar otros sistemas del contexto, se hace necesario un enfoque el cual también sea capaz de medir el beneficio del modelo tanto para las instituciones como para el docente. La perspectiva teórica predominante para su implantación es de corte constructivista, pero se señala que el blended learning debe también apoyarse y tomar fundamentos de otras teorías para su implementación.

Para concluir, otras líneas de investigación urgentes para el blended learning son aquellas relacionadas con sus fundamentos conceptuales, medición de su eficacia desde una perspectiva sistémica, una detección de necesidades de capacitación específica en habilidades y competencias tanto didácticas como tecnológicas para los docentes, específicamente en contextos híbridos; la indagación de los fundamentos de otras teorías como el cognitivismo y el conectivismo para el diseño de estrategias didácticas de blended learning su repercusión en el proceso de enseñanza aprendizaje, así como bondades y limitaciones de los modelos existentes en diversos contextos, con el fin de contar referencias válidas tanto para su implementación como para su mejora.

\section{Agradecimientos}

Se agradece al Consejo Nacional de Ciencia y Tecnología de México por el apoyo brindado para la elaboración de este documento.

\section{Referencias}

Baeza-Correa, J. (Ed.). (2008). Drogas en América latina. Estado del arte en estudios de toxicomanía en Argentina, Brasil, Colombia, Chile y Ecuador. UCSH.

Breen, P. (2018). Blended Learning's Consistent Presence. In: Developing Educators for The Digital Age: A Framework for Capturing Knowledge in Action. 127142. University of Westminster Press. http://www.jstor.org/st able/j.ctv5vddjh.12

Cabero-Almenara, J., Roig-Vila, R. \& Mengual-Andrés, S. (2017). Conocimientos tecnológicos, pedagógicos y disciplinares de los futuros docentes. Según el modelo TPACK. Digital Education Review. (32), 7384. https://doi.org/10.1344/der.2017.32.73-84

Cabero, J., \& Román, P. (2008). E-actividades. Un referente básico para la información en Internet. Editorial MAD.

Chris, A., Moldovan, A., Murphy, L., Pathak, P., \& Muntean, C. (2018). Investigating Flipped Classroom and Problem-based Learning in a Programming Module for Computing Conversion Course. Journal of Educational Technology \& Society, 21(4), 232-247. http://www.jstor.org/stable/265115515

Cuesta-Medina, L. (2018). Blended learning: Deficits and prospects in higher education. Australasian Journal of Educational Technology, 34(1). https://doi.org/10.14742/ajet.3100

Cuevas, R., Feliciano, A., Miranda, A. y Catalán, A. (2015). Corrientes teóricas sobre aprendizaje combinado en educación. Revista Iberoamericana de Ciencias. 2(1), 75-84. http://www.reibci.org/

Gallou, E., \& Abrahams, P. (2018). Creating space for active learning: (Opportunities from) using technology in research-based education. In Tong V., Standen A., \& Sotiriou M. (Eds.), Shaping Higher Education with Students: Ways to Connect Research and Teaching. 165-175. London: UCL Press. www.jstor.org/stable/j.ctt21c4tcm.27

García-Aretio, L. (2018). Blended Learning y la convergencia entre la educación presencial y a distancia. Revista Iberoamericana de Educación a Distancia, 21(1), 9-22. http://revistas.uned.es/index.php/ried/article/view/19 683 


\section{Acercamiento a la Discusión Académica sobre Blended Learning.}

Graham, C. R. (2004). Blended learning Systems: Definition, current trends and future directions, Handbook of blended learning: Global Perspectives, local designs, 2004.

Gunyou, J. (2015). I Flipped My Classroom: One Teacher's Quest to Remain Relevant. Journal of Public Affairs Education, 21(1), 13-24. www.jstor.org/stable/24369701

Hoyos-Botero, C. (2000). Un modelo para investigación documental: guía teórico-práctica sobre construcción de Estados del Arte con importantes reflexiones sobre la investigación. Señal Editora.

Johnson, L., Becker, S.A., Estrada, V., \& Freeman, A. NMC Horizon Report: 2015 Higher (2015). Education.Texas: Edition Austin.

Llorente-Cejudo, M.C. \& Cabero-Almenara, J. (2008). Del E-Learning al Blended Learning: nuevas acciones educativas. Revista de Nuevas Tecnologías y Sociedad (51). https://dialnet.unirioja.es/servlet/articulo? $\operatorname{codigo}=25$ 66563

Londoño-Palacio, O. L., Maldonado-Granados, L. F., \& Calderón-Villafánez, L. C. (2016). Guía para construir estados del arte. Bogotá: International Corporation of Network of Knowledge. http://iconk.org/docs/guiaea.pdf

Prohorets, E. \& Plekhanova, M. (2014) Interaction intensity levels in blended learning environment. Elsevier, 174.

Rodríguez, R., \& Espinoza, L. (2017). La modalidad Blended learning como alternativa de un ambiente de aprendizaje innovador. Revista Iberoamericana de las Ciencias Computacionales e Informática, 6(11), 1-21.

Martín-García, A., Serrano, M., \& Gómez, M. (2014). Fases y clasificación de adoptantes de blended learning en contextos universitarios. Aplicación del análisis CHAID. Revista Española De Pedagogía, 72(259), 457-476. http://www.jstor.org/stable/24726632

Medina-Montoya, S. I. (2004). Función social del psicólogo clínico: estado del arte. (Trabajo de grado inédito). Universidad San Buenaventura, Medellín.

Núñez-Barriopedro, E., Monclúz, I.M., \& Ravina-Ripoll, R. (2019). El impacto de la utilización de la modalidad Blended learning en la educación superior. Alteridad, 14(1), 26-39. https://dx.doi.org/10.17163/alt.v14n1.2019.02

Seraji, F., Attaran, M. \& Azizi, S.M. (2019). Blended Learning Researches in Iran: Several Fundamental Criticism. Digital Education Review. Diciembre (36), 190-206. https://doi.org/10.1344/der.2019.36.190206

Siemens, G. (2004). Connectivism. A learning Theory for the Digital Age. Traducida por Diego Leal Fonseca en 2007.
https://docs.google.com/document/d/1ZKuAzdx119 IDgeC1E_XSmPTOk6Gu1K3SEvXtduG3ge/edi

Singh, H. (2003). Building effective blended learning programs. Education and Technology, 43(6), 51-54.

Shea, J., Joaquin, M., \& Wang, J. (2016). Pedagogical Design Factors That Enhance Learning in Hybrid Courses: A Contribution to Design-Based Instructional Theory. Journal of Public Affairs Education, 22(3), 381-397. http://www.jstor.org/stable/44114744

Sobrino, Á. (2014). Aportaciones del conectivismo como modelo pedagógico post-constructivista. Propuesta Educativa, (42), 39-48. https://www.redalyc.org/comocitar.oa?id=40304171 3005

Solórzano-Martínez, F. \& Garcia-Martinez, A. (2016). Fundamentos del aprendizaje en red desde el conectivismo y la teoría de la actividad. Revista Cubana de Educación Superior, (3), 98-111. http://scielo.sld.cu/scielo.php?script=sci abstract\&p id $=$ S0257-43142016000300008

Spring, K. J., Graham, C.R., \& Hadlock, C.A. (2016). The current landscape of international blended learning. International Journal of Technology Enhanced Learning, 8(1), 84-102.

Straus, S., Shanley, M., Lytell, M., Crowley, J., Bana, S., Clifford, M., \& Leuschner, K. (2013). Enhancing Critical Thinking Skills for Army Leaders Using Blended-Learning Methods. RAND Corporation. http://www.jstor.org/stable/10.7249/j.ctt4cgdsr

Trentin, G. (2016). Always-on Education and Hybrid Learning Spaces. Educational Technology, 56(2), 31-37. http://www.jstor.org/stable/44430457

Vásquez, M., Hidalgo, J., \& Vergara-Lozano, V. (2017). Evaluación de la efectividad de un sistema blended learning. Revista de Investigación en Tecnologías de la Información, 5(9), 1-6. http://www.riti.es/ojs2018/inicio/index.php/riti/articl e/view/17

Wang, Y., Han, X., \& Yang, J. (2015). Revisiting the Blended Learning Literature: Using a Complex Adaptive Systems Framework. Journal of Educational Technology \& Society, 18(2), 380-393. http://www.jstor.org/stable/jeductechsoci.18.2.380

Wang, Q., Quek, C. L., \& Hu, X. (2017). Designing and Improving a Blended Synchronous Learning Environment: An Educational Design Research. The International Review of Research in Open and Distributed Learning, 18(3). https://doi.org/10.19173/irrodl.v18i3.3034

Wengreen, H., Dimmick, M. \& Israelsen, M. (2015). Evaluation of a Blended Design in a Large General Education Nutrition Course. NACTA Journal, 59(1), 70-74. http://www.jstor.org/stable/nactajournal.59.1.70

Zabala, A., \& Arnau, L. (2014). Métodos para la enseñanza de las competencias. Graó 